\title{
Correction to: Comparison of in vitro fertilisation/intracytoplasmic sperm injection on live birth rates in couples with non-male factor infertility and advanced maternal age
}

\author{
Nicole O. McPherson ${ }^{1,2,3,4}$ (D) Andrew D. Vincent ${ }^{1,4,5} \cdot$ Leanne Pacella-Ince $^{2,3} \cdot$ Kelton Tremellen $^{3,6}$ \\ Published online: 10 February 2021 \\ (C) Springer Science+Business Media, LLC, part of Springer Nature 2021
}

\section{Correction to: Journal of Assisted Reproduction and Genetics} https://doi.org/10.1007/s10815-020-02026-8

The original article unfortunately contained a mistake in the first line of the Conclusion section.

"In couples with advanced maternal age and non-male factor infertility, it appears that standard IVF insemination increased the chance of a live birth at least 9-fold compared with ICSI Insemination"

When it should read

"In couples with advanced maternal age and non-male factor infertility, it appears that standard IVF insemination increased the chance of a live birth at least 2-fold compared with ICSI insemination"

The original article has been corrected.

Publisher's note Springer Nature remains neutral with regard to jurisdictional claims in published maps and institutional affiliations.

The online version of the original article can be found at https://doi.org/ 10.1007/s10815-020-02026-8

Nicole O. McPherson

nicole.mcpherson@adelaide.edu.au

1 Freemasons Center for Male Health and Wellbeing, The University of Adelaide, Adelaide, SA 5005, Australia

2 Robinson Research Institute, The University of Adelaide, Adelaide, SA 5005, Australia

3 Repromed, Dulwich, Adelaide, SA 5065, Australia

4 Adelaide Health and Medical Science, The University of Adelaide, Adelaide, SA 5005, Australia

5 South Australian Health and Medical Research Institute, Adelaide, SA 5001, Australia

6 Department of Obstetrics Gynaecology and Reproductive Medicine, Flinders University, Adelaide, Adelaide, SA 5042, Australia 